\title{
The Influence of Local Increase of Stress on Eigenvalues for a Simply Supported Beam
}

\author{
S. KASPRZYK AND R. MARCZUK* \\ AGH University of Science and Technology, Department of Mechanics and Vibroacoustics, \\ Al. A. Mickiewicza 30, 30-059 Krakow, Poland
}

Local increase of bending stress in a beam may be caused by a decrease of the cross-section (fracture) or a local increase of bending torque. The increase of stress issues from the well known interdependence between the stress, the bending torque and the sectional modulus. The work presents a derivation of differential equations for eigenfunctions in both cases. Knowing the eigenfunctions and boundary conditions we determine a system of algebraic equations for the eigenvalues that are different from the eigenvalues of the beam without local stress disturbances. Two computational models of local increase of stress were constructed: with fracture and with local increase of bending torque.

DOI: 10.12693/APhysPolA.125.A-169

PACS: 46.70.De, 46.40.-f

\section{Introduction}

The subjects raised in this paper was discussed in many articles, e.g. [1-3]. Applicability of the separation of variables method in the linear discrete-continuous systems in distribution sense can be founded in [1]. Deriving a differential equation of transverse vibrations of a beam with a local change of stiffness and it solution based on theory of distribution is presented in [2]. In Ref. [3] was analysed system with stochastic impulses acting in on it in the class of generalized functions.

In this paper, using generalized functions approach were constructed two computational models of local increase of stress. The local increase of bending stress in Euler's beam may be caused by a decrease of its transverse section (e.g., due to a fracture) or by a local increase of the bending torque. The increase of the stress results from the well-known interdependence between the stress, the bending torque and the bending strength index. The local increase of the bending torque may be caused either external or internal forces [4].

In the case under consideration it will be caused by the internal forces of a certain discrete-continuous system. A model of this system was discussed in [52]. The purpose of this work is to solve the eigenproblems in both cases mentioned above. To this end, a mathematical model of the initial-boundary problems is presented. It is shown that the method of separation of variables can be successfully applied for the case with structural dumping factor, $\alpha_{0}$ different from zero.

\section{Formulation of the problem}

The differential equation describing transverse vibrations of a beam has a form (Ref. [6], p. 173 and 87)

*corresponding author; e-mail: marczuk@agh.edu.pl

$$
\frac{\partial^{2} M(x)}{\partial x^{2}}+\rho F(x) \frac{\partial^{2} u(x, t)}{\partial t^{2}}=q(x, t), \quad \rho>0,
$$
where

$$
\begin{aligned}
& M(x)=E J(x)\left(\frac{\partial^{2} u(x, t)}{\partial x^{2}}+\frac{\alpha_{0}}{E} \frac{\partial^{3} u(x, t)}{\partial x^{2} \partial t}\right), \\
& \alpha_{0}>0, E>0,
\end{aligned}
$$

and $u(x, t)$ is a beam deflection at point $x$ and at a moment $t$ of time; $\alpha_{0}$ is a structure dumping factor; $E$ denotes Young's modulus; $J(x)$ is the cross section moment of inertia; $F(x)$ is the cross section area, $\varrho$ is a density of beam's material.

We assume that functions $M(x), J(x)$ and $F(x)$ are at least twice differentiable in the classical sense. Intending to derive the equation of a beam with a fracture, we must weaken the assumption about differentiability of $M(x)$, $J(x)$ and $F(x)$.

The boundary conditions for (1) have the form: (the free end of beam)

$$
u(0, t)=u(l, t)=0, \frac{\partial^{2} u(0, t)}{\partial x^{2}}=\frac{\partial^{2} u(l, t)}{\partial x^{2}}=0
$$

and the initial conditions are:

$$
u(x, 0)=\Phi_{0}(x), \frac{\partial u(x, 0)}{\partial t}=\Phi_{1}(x) .
$$

\section{Cases of the local increase of stress}

\subsection{The case of a fracture}

We shall seek solutions of the above mentioned initialboundary problems in the class of generalized functions (differentiation in the distribution sense) [7-9]. As for functions $J(x)$ and $F(x)$ we make the following assumptions:

$$
\begin{aligned}
& J(x)=J_{0}\left(1+\gamma_{1} H\left(x_{1}, x_{2}\right)\right), \quad x \in\langle 0, l\rangle, \\
& F(x)=F_{0}\left(1+\gamma_{2} H\left(x_{1}, x_{2}\right)\right), \\
& x \in\langle 0, l\rangle, x_{0} \in\langle 0, l\rangle,
\end{aligned}
$$


$H\left(x-x_{2}\right), x_{1}=x_{0}-\varepsilon, x_{2}=x_{0}+\varepsilon, \varepsilon>0, H(x-a)=1$ for $x>a, H(x-a)=\frac{1}{2}$ for $x=a, H(x-a)=0$ for $x<a, J_{0}$ is the cross-section moment of inertia and $F_{0}$ is the cross section area.

Due to the form of functions Eqs. (5) and (6) we will write (2) in the form (Refs. [10] p. 173, [6] p. 94)

$$
M(x)=E \frac{\partial}{\partial x}\left[J(x)\left(\frac{\partial u}{\partial x}+\frac{\alpha_{0}}{E} \frac{\partial^{2} u}{\partial x \partial t}\right)\right] .
$$

Putting Eq. (7) into Eq. (1) and taking Eqs. (5) and (6) into account we obtain:

$$
\begin{gathered}
E J_{0} \frac{\partial^{3}}{\partial x^{3}}\left\{\left[1+\gamma_{1} H\left(x_{1}, x 2\right)\right]\left(\frac{\partial u}{\partial x}+\frac{\alpha_{0}}{E} \frac{\partial^{2} u}{\partial x \partial t}\right)\right\}+ \\
\rho F_{0}\left[1+\gamma_{2} H\left(x_{1}, x_{2}\right)\right] \frac{\partial^{2} u}{\partial t^{2}}=q(x, t)
\end{gathered}
$$

Let consider Eq. (6) with boundary conditions Eqs. (3) and (4) describing the initial-boundary problem for Euler's beam assuming a fracture at the point $x_{0}$. To solve this problem it is convenient to use the method of separation of variables:

$$
u(x, t)=X(x) T(t)
$$

Inserting Eq. (9) in Eqs. (6) and Eq. (3) for $q(x, t) \equiv 0$, after standard transformations we obtain

$$
\begin{aligned}
& -\frac{E J_{0} \frac{\mathrm{d}^{3}}{\mathrm{~d} x^{3}}\left\{\left[1+\gamma_{1} H\left(x_{1}, x_{2}\right)\right] X^{\prime}(x)\right\}}{\rho F_{0}\left(1+\gamma_{2} H\left(x_{1}, x_{2}\right)\right) X(x)}=\frac{\ddot{T}}{T+\frac{\alpha_{0}}{E} \dot{T}}= \\
& -\omega^{2}
\end{aligned}
$$

and from Eq. (3) it follows that

$$
X(0)=X(l)=0, X^{\prime \prime}(0)=X^{\prime \prime}(l)=0 .
$$

$$
\begin{aligned}
& \text { Hence } \\
& \begin{array}{l}
\ddot{T}+\omega^{2} \frac{\alpha_{0}}{E} \dot{T}+\omega^{2} T=0 \\
E J_{0} \frac{\mathrm{d}^{3}}{\mathrm{~d} x^{3}}\left\{\left[1+\gamma_{1} H\left(x_{1}, x_{2}\right)\right] X^{\prime}(x)\right\} \\
\quad+\rho F_{0}\left(1+\gamma_{2} H\left(x_{1}, x_{2}\right)\right) X(x) \omega^{2}=0 .
\end{array}
\end{aligned}
$$

Function Eq. (5) does not involve structural damping constant $\alpha_{0}$ so eigenfrequencies (eigenvalues) do not depend on $\alpha_{0}$. Equation (13) and conditions Eq. (11) represent the eigenproblem of a beam with a fracture at the point $x_{0}$. Differentiated three times equation Eq. (13) will take the form (Refs. [9] p. 76, [6] p. 123):

$$
\begin{aligned}
& E J_{0}\left\{-\gamma_{1}\left(X^{\prime}\left(x_{2}\right) \delta^{\prime \prime}\left(x-x_{2}\right)-X^{\prime}\left(x_{1}\right) \delta^{\prime \prime}\left(x-x_{1}\right)\right)\right. \\
& \quad-\gamma_{1}\left(X^{\prime \prime}\left(x_{2}\right) \delta^{\prime}\left(x-x_{2}\right)-X^{\prime \prime}\left(x_{1}\right) \delta^{\prime}\left(x-x_{1}\right)\right. \\
& \quad-\gamma_{1}\left(X^{\prime \prime \prime}\left(x_{2}\right) \delta\left(x-x_{2}\right)-X^{\prime \prime \prime}\left(x_{1}\right) \delta\left(x-x_{1}\right)\right) \\
& \left.\quad+X^{\prime \prime \prime \prime}(x)+\gamma_{1} H\left(x_{1}, x_{2}\right) X^{\prime \prime \prime \prime}(x)\right\} \\
& \quad-\rho F_{0}\left(1+\gamma_{2} H\left(x_{1}, x_{2}\right)\right) X(x) \omega^{2}=0 .
\end{aligned}
$$

Lemma 1. For sufficiently small $\varepsilon>0$ we have:

$$
\left\{\begin{array}{l}
X^{\prime}\left(x_{0}+\varepsilon\right) \delta^{\prime \prime}\left(x, x_{0}+\varepsilon\right)-X^{\prime}\left(x_{0}-\varepsilon\right) \delta^{\prime \prime}\left(x, x_{0}-\varepsilon\right) \cong \\
\left(X^{\prime}\left(x_{0}+\varepsilon\right)-X^{\prime}\left(x_{0}-\varepsilon\right)\right) \delta^{\prime \prime}\left(x-x_{0}\right), \\
X^{\prime \prime}\left(x_{0}+\varepsilon\right) \delta^{\prime}\left(x, x_{0}+\varepsilon\right)-X^{\prime \prime}\left(x_{0}-\varepsilon\right) \delta^{\prime}\left(x, x_{0}-\varepsilon\right) \cong \\
\quad\left(X^{\prime \prime}\left(x_{0}+\varepsilon\right)-X^{\prime \prime}\left(x_{0}-\varepsilon\right)\right) \delta^{\prime}\left(x-x_{0}\right), \\
X^{\prime \prime \prime}\left(x_{0}+\varepsilon\right) \delta\left(x, x_{0}+\varepsilon\right)-X^{\prime \prime \prime}\left(x_{0}-\varepsilon\right) \delta\left(x, x_{0}-\varepsilon\right) \cong \\
\left(X^{\prime \prime \prime}\left(x_{0}+\varepsilon\right)-X^{\prime \prime \prime}\left(x_{0}-\varepsilon\right)\right) \delta\left(x-x_{0}\right), \\
H\left(x_{1}, x_{2}\right) X(x)=\left(H\left(x_{0}-\varepsilon\right)-H\left(x_{0}+\varepsilon\right)\right) X(x) \cong \\
X\left(x_{0}\right) \delta\left(x-x_{0}\right),
\end{array}\right.
$$

where: $\delta^{\prime \prime}$ is the second derivative of $\delta$ and $X^{\prime}, X^{\prime \prime}, X^{\prime \prime \prime}$ are derivatives of function $X(x)$ (Eq. (9)).

Lemma 2. For sufficiently small $\eta>0$

$$
\begin{aligned}
& \left(H\left(x_{0}-\eta\right)-H\left(x_{0}+\eta\right)\right) X^{\prime \prime \prime \prime}(x)= \\
& \frac{\gamma_{1}}{1+\gamma_{1}} \sigma_{3}\left(x_{0}\right) \delta\left(x-x_{0}\right),
\end{aligned}
$$

where $-1<\gamma_{1} \leq 0, \sigma_{3}$ is defined in Eq. (24).

The known formulae (Ref. [9] p. 76, 85) are used in the proofs of Lemma 1 and 2:

$$
\begin{aligned}
& \alpha(x) \delta\left(x-x_{0}\right)=\alpha\left(x_{0}\right) \delta\left(x-x_{0}\right), \\
& \alpha(x) \delta^{\prime}\left(x-x_{0}\right)=\alpha^{\prime}\left(x_{0}\right) \delta\left(x-x_{0}\right)+\alpha\left(x_{0}\right) \delta^{\prime}\left(x-x_{0}\right), \\
& \alpha\left(x_{0}\right) \delta^{\prime \prime}\left(x-x_{0}\right)=\alpha^{\prime}\left(x_{0}\right) \delta\left(x-x_{0}\right) \\
& \quad \times-2 \alpha^{\prime}\left(x_{0}\right) \delta^{\prime}\left(x-x_{0}\right)+\alpha\left(x_{0}\right) \delta^{\prime \prime}\left(x-x_{0}\right),
\end{aligned}
$$

where $\alpha$ is of an appropriate regularity. For sufficiently small $\varepsilon>0$, for any test function $\varphi$ we have $\varphi^{\prime \prime}\left(x_{0} \pm \varepsilon\right) \cong$ $\varphi^{\prime \prime}\left(x_{0}\right) \pm \varphi^{\prime \prime \prime}\left(x_{0}\right) \cdot \varepsilon$, while $\varphi^{\prime \prime \prime}\left(x_{0}\right) \cdot \varepsilon \rightarrow 0$ when $\varepsilon \rightarrow 0$.

The proof of the first formula in Lemma 1, Eq. (15)

Multiplying both sides of the aforementioned equation by test function $\varphi(x)$ and integrating, we obtain

$$
\begin{aligned}
& \int_{\mathbb{R}}\left(X^{\prime}\left(x_{0}+\varepsilon\right) \delta^{\prime \prime}\left(x, x_{0}+\varepsilon\right)-X^{\prime}\left(x_{0}-\varepsilon\right)\right. \\
& \left.\quad \times \delta^{\prime \prime}\left(x, x_{0}-\varepsilon\right)\right) \varphi(x) \mathrm{d} x \cong \int_{\mathbb{R}}\left(X^{\prime}\left(x_{0}+\varepsilon\right)\right. \\
& \left.\quad-X^{\prime}\left(x_{0}-\varepsilon\right) \delta^{\prime \prime}\left(x, x_{0}+\varepsilon\right)\right) \delta^{\prime \prime}\left(x-x_{0}\right) \varphi(x) \mathrm{d} x .
\end{aligned}
$$

Using the equalities (17) in (18) we obtain the following identity:

$$
\begin{gathered}
\left(X^{\prime}\left(x_{0}+\varepsilon\right)-X^{\prime}\left(x_{0}-\varepsilon\right)\right) \varphi^{\prime \prime}\left(x_{0}\right) \equiv \\
\left(X^{\prime}\left(x_{0}+\varepsilon\right)-X^{\prime}\left(x_{0}-\varepsilon\right)\right) \varphi^{\prime \prime}\left(x_{0}\right) .
\end{gathered}
$$

As it folllows from Eq. (23) function $X^{\prime}(x)$ is continuous in the neighbourhood of $x_{0}$ and Eq. (19) holds for every $\varphi^{\prime \prime}$, q.e.d.

The remaining equations Eq. (15) can be proved in the same way.

The proof of Lemma 2

The proof of Eq. (16) consists in using the formula: $X^{(i)}\left(x_{0} \pm \varepsilon\right) \cong X^{(i)}\left(x_{0}\right) \pm X^{(i+1)}\left(x_{0}\right) \varepsilon,(i=0,1,2,3)$. Deriving equations for eigenvalues $\lambda$, we execute a standard procedure using the boundary conditions Eq. (3), the solution of $X(x)$ in the form Eq. (23), the derivatives $X^{(i)}(x),(i=1,2)$, and the equation Eq. (24).

We multiply both sides of the equation Eq. (16) by the 
test function $\varphi(x), H\left(x_{1}, x_{2}\right)=H\left(x_{0}-\varepsilon\right)-H\left(x_{0}+\varepsilon\right)$ and integrate the result in the interval $\left(x_{0}-\varepsilon, x_{0}+\varepsilon\right)$. Then we use the first three equations (17) and obtain

$$
\begin{aligned}
& \int_{x_{0}-\varepsilon}^{x_{0}+\varepsilon}\left(H\left(x_{1}, x_{2}\right)\left(1+\gamma_{1}\right) X^{\prime \prime \prime \prime}(x)-\gamma_{1} 2 \sigma_{3}\left(x_{0}\right)\right) \\
& \quad \times \delta\left(x-x_{0}\right) \varphi(x) \mathrm{d} x \cong 0 .
\end{aligned}
$$

As the component

$$
\int_{x_{0}-\varepsilon}^{x_{0}+\varepsilon} X(x) H\left(x_{1}, x_{2}\right) \varphi(x) \mathrm{d} x \cong 0
$$

is very small and therefore can be neglected, whereas we know that Eq. (20) holds for every $\varphi$, which means that

$$
H\left(x_{1}, x_{2}\right) X^{\prime \prime \prime \prime}(x) \cong \frac{2 \gamma_{1}}{1+\gamma_{1}} \sigma_{3}\left(x_{0}\right) \delta\left(x-x_{0}\right),
$$

q.e.d.

Due to Eqs. (15) and (16), equation (14) will take the form

$$
\begin{aligned}
& X^{\prime \prime \prime \prime}-\lambda^{4} X=-\gamma_{1}\left[\sigma_{1}\left(x_{0}\right) \delta^{\prime \prime}\left(x-x_{0}\right)+\sigma_{2}\left(x_{0}\right)\right. \\
& \left.\quad \times \delta^{\prime}\left(x-x_{0}\right)+\frac{1}{1-\gamma_{1}} \sigma_{3}\left(x_{0}\right) \delta\left(x-x_{0}\right)\right],
\end{aligned}
$$

where: $\lambda^{4}=\frac{\rho F_{0} \omega^{2}}{E J_{0}}, \sigma_{i}\left(x_{0}\right)=X^{(i)}\left(x_{0}+\epsilon\right)-X^{(i)}\left(x_{0}-\right.$ $\epsilon), i=1,2,3$.

Solution of Eq. (22) can be expressed by the formula $X(x)=P \cos \lambda x+Q \cos \lambda x+R \cosh \lambda x+S \sinh \lambda x$

$$
\begin{aligned}
& +\gamma_{1}\left\{\frac{\sigma_{1}\left(x_{0}\right)}{2 \lambda}\left[\sinh \lambda\left(x-x_{0}\right)+\sin \lambda\left(x-x_{0}\right)\right]+\right. \\
& +\frac{\sigma_{2}\left(x_{0}\right)}{2 \lambda^{2}}\left[\cosh \lambda\left(x-x_{0}\right)-\cos \lambda\left(x-x_{0}\right)\right]+ \\
& \left.+\frac{1}{1+\gamma_{1}} \frac{\sigma_{3}\left(x_{0}\right)}{2 \lambda^{3}}\left[\sinh \lambda\left(x-x_{0}\right)-\sin \lambda\left(x-x_{0}\right)\right]\right\} \\
& \times H\left(x-x_{0}\right),
\end{aligned}
$$

where

$$
\begin{aligned}
& \sigma_{1}\left(x_{0}\right)=\frac{X_{0}^{\prime}\left(x_{0}\right)}{1-\gamma_{1} d_{1}}, \sigma_{2}\left(x_{0}\right)=\frac{X_{0}^{\prime \prime}\left(x_{0}\right)}{1-\gamma_{1} d_{2}} \sigma_{3}\left(x_{0}\right)= \\
& \frac{1+\gamma_{1}}{1+\gamma_{1}\left(1-d_{3}\right)} X_{0}^{\prime \prime \prime}\left(x_{0}\right),
\end{aligned}
$$

and $X_{0}(x)=P \cos \lambda x+Q \sin \lambda x+R \cosh \lambda x+S \sinh \lambda x$ $-1<\gamma_{1} \leq 0$.

By Eq. (11), $P=R=0$ while the remaining constants $Q$ and $S$ satisfy the equation

$$
\left[\begin{array}{l}
a_{11} a_{12} \\
a_{21} a_{22}
\end{array}\right]\left[\begin{array}{l}
Q \\
S
\end{array}\right]=\left[\begin{array}{l}
0 \\
0
\end{array}\right],
$$

where:

$$
\begin{aligned}
& a_{11}=\sin \lambda l+\frac{\gamma_{1}}{2 \lambda\left(1-\gamma_{1}\right)}\left\{\operatorname { c o s } \lambda x _ { 0 } \left[\sinh \lambda\left(l-x_{0}\right)\right.\right. \\
& \left.+\sin \lambda\left(l-x_{0}\right)\right]-\frac{1}{\lambda} \sin \lambda x_{0}\left[\cosh \lambda\left(l-x_{0}\right)\right. \\
& \left.-\cos \lambda\left(l-x_{0}\right)\right]+\frac{1}{\lambda^{2}}\left(1-\gamma_{1}\right) \cos \lambda x_{0}
\end{aligned}
$$

$$
\begin{aligned}
\times & {\left.\left[\sinh \lambda\left(l-x_{0}\right)-\sin \lambda\left(l-x_{0}\right)\right]\right\}, } \\
a_{12} & =\sinh \lambda l+\frac{\gamma_{1}}{2 \lambda\left(1-\gamma_{1}\right)}\left\{\operatorname { c o s h } \lambda x _ { 0 } \left[\sinh \lambda\left(l-x_{0}\right)\right.\right. \\
& \left.+\sin \lambda\left(l-x_{0}\right)\right]+\frac{1}{\lambda} \sinh \lambda x_{0}\left[\cosh \lambda\left(l-x_{0}\right)\right. \\
- & \left.\cos \lambda\left(l-x_{0}\right)\right]+\frac{1}{\lambda^{2}}\left(1-\gamma_{1}\right) \cosh \lambda x_{0} \\
\quad \times & {\left.\left[\sinh \lambda\left(l-x_{0}\right)-\sin \lambda\left(l-x_{0}\right)\right]\right\}, } \\
a_{21} & =-\sin \lambda l+\frac{\gamma_{1}}{2 \lambda\left(1-\gamma_{1}\right)} \\
& \times\left\{\cos \lambda x_{0}\left[\sinh \lambda\left(l-x_{0}\right)-\sin \lambda\left(l-x_{0}\right)\right]\right. \\
& -\frac{1}{2 \lambda} \sin \lambda x_{0}\left[\cosh \lambda\left(l-x_{0}\right)-\cos \lambda\left(l-x_{0}\right)\right] \\
+ & \left.\frac{1}{\lambda^{2}}\left(1-\gamma_{1}\right)\left[\sinh \lambda\left(l-x_{0}\right)+\sin \lambda\left(l-x_{0}\right)\right]\right\}, \\
a_{22} & =\sinh \lambda l+\frac{\gamma_{1}}{2 \lambda\left(1-\gamma_{1}\right)}\left\{\operatorname { c o s h } \lambda x _ { 0 } \left[\sinh \lambda\left(l-x_{0}\right)\right.\right. \\
- & \left.\sin \lambda\left(l-x_{0}\right)\right]+\frac{1}{\lambda} \sinh \lambda x_{0}\left[\cosh \lambda\left(l-x_{0}\right)\right. \\
+ & \left.\cos \lambda\left(l-x_{0}\right)\right]+\frac{1}{\lambda^{2}}\left(1-\gamma_{1}\right) \cosh \lambda x_{0} \\
\times & {\left.\left[\sinh \lambda\left(l-x_{0}\right)+\sin \lambda\left(l-x_{0}\right)\right]\right\} . }
\end{aligned}
$$

The equation for eigenvalues takes the form:

$$
\operatorname{det}\left[\begin{array}{l}
a_{11} a_{12} \\
a_{21} a_{22}
\end{array}\right]=0 .
$$

From the form of the terms $a_{i j}$ it is clear that eigenvalues $\lambda$ depend on $\gamma_{1}$. The adopted model will be correct if

$$
0<\lambda_{n}\left(\gamma_{1}\right)<\frac{\pi n}{l}, n \in \mathbb{N},
$$

where $\frac{\pi n}{l}$ are eigenvalues of beam without fractures.

\subsection{The case of a local increase of the bending torque caused by the internal forces}

In this case we will use a discrete-continuous system presented in Fig. 1, where $q(x, t)$ is a generalized external load; elasticity coefficients $k$ and $k_{1}$ must not be too high, because otherwise local stiffening would take place. Since the problem of eigenvalues is considered, structural damping of the system is omitted.

If the internal forces in the springs satisfy the equation

$$
k u\left(x_{0}-\varepsilon, t\right)+k u\left(x_{0}+\varepsilon, t\right)=k_{1}\left(y(t)-u\left(x_{0}, t\right)\right),
$$

$$
0<\epsilon<1 \text {, }
$$

these forces do not change the response of the supports.

The mathematical description of the initial-boundary problem associated with the model in Fig. 1 is presented by the system of equations (Ref. [6] p. 102) 


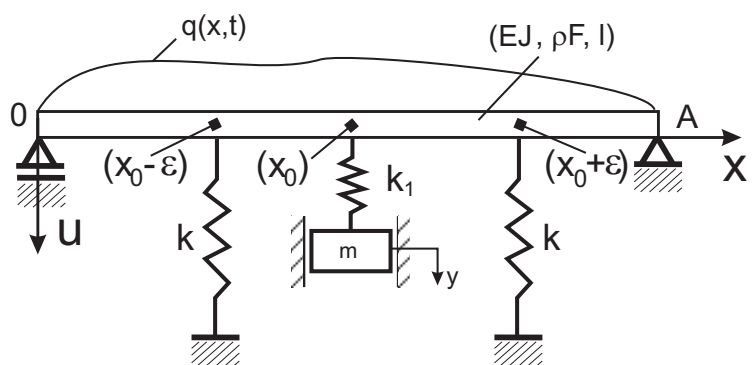

Fig. 1. Model of the system.

$$
\left\{\begin{array}{l}
E J \frac{\partial^{4} u}{\partial x^{4}}+\varrho F \frac{\partial^{2} u}{\partial t^{2}}-k_{1}\left(y-u\left(x_{0} t\right)\right) \delta\left(x-x_{0}\right) \\
\quad+k u\left(x_{1}, t\right) \delta\left(x-x_{1}\right)+k u\left(x_{2}, t\right) \delta\left(x-x_{2}\right) \\
\quad=q(x, t), \\
m \ddot{y}+k_{1}\left(y-u\left(x_{0}, t\right)\right)=0 \\
x_{1}=x_{0}-\varepsilon, x_{2}=x_{0}-\varepsilon, \\
\quad 0<\varepsilon \text { arbitrarily small, }
\end{array}\right.
$$

with the boundary conditions

$$
\begin{aligned}
& u(0, t)=u(l, t)=0, \\
& \frac{\partial^{2} u(o, t)}{\partial x^{2}}=\frac{\partial^{2} u(l, t)}{\partial x^{2}}=0,
\end{aligned}
$$

and the initial conditions

$$
u(x, 0)=\varphi_{0}(x), \frac{\partial u(x, 0)}{\partial t}=\varphi_{1}(x) .
$$

Let us assume that

$$
u(x, t)=X(x) T(t), y(t)=A T(t) .
$$

Due to Eq. (31), after standard transformations, equations Eq. (29), with $q(x, t) \equiv 0$, conditions Eq. (32) and condition Eq. (28) will take the form

$$
\begin{aligned}
& \ddot{T}+\omega^{2} T=0, \\
& X^{\prime \prime \prime \prime}-\lambda^{4} X=-\frac{k_{1}}{E J}\left(A-X\left(x_{0}\right) \delta\left(x-x_{0}\right)\right) \\
& \quad+\frac{k}{E J}\left[X\left(x_{1}\right) \delta\left(x-x_{1}\right)+X\left(x_{2}\right) \delta\left(x-x_{2}\right)\right],
\end{aligned}
$$

where

$$
\begin{aligned}
& \lambda^{4}=\frac{\varrho F \omega^{2}}{E J}, \quad k_{1} \frac{A-X\left(x_{0}\right)}{m A}=\omega^{2}, \text { or } \\
& A=\frac{\omega_{0}^{2} X\left(x_{0}\right)}{\omega_{0}^{2}-\omega^{2}}, \omega_{0}^{2}=\frac{k_{1}}{m}, \\
& X(0)=X(l)=0, X^{\prime \prime}(0)=X^{\prime \prime}(l)=0,
\end{aligned}
$$

wherein was assumed that

$$
\begin{aligned}
& X\left(x_{1}\right)+X\left(x_{2}\right)=2 X\left(x_{0}\right), x_{1}=x_{0}-\varepsilon, \\
& \quad x_{2}=x_{0}+\varepsilon, \varepsilon>0-- \text { arbitrarily small. }
\end{aligned}
$$

From Eqs. (35), (37), and the formula Eq. (28) it follows that

$$
\frac{k}{k_{1}}=-\frac{1}{2} \frac{\omega^{2}}{\omega_{0}^{2}+\omega^{2}} .
$$

The above formula represents the relationship between $k, k_{1}$, and $m$. Reasoning along the same line as in the proof of Lemma 1, we shall obtain

$$
\begin{gathered}
X\left(x_{0}-\varepsilon\right) \delta\left(x_{0}-\varepsilon\right)+X\left(x_{0}+\varepsilon\right) \delta\left(x_{0}+\varepsilon\right) \cong \\
\left(X\left(x_{0}-\varepsilon\right)+X\left(x_{0}+\varepsilon\right)\right) \delta\left(x-x_{0}\right) .
\end{gathered}
$$

Due to Eqs. (37) and (39), Eq. (34) will assume the form

$$
\begin{aligned}
& X^{\prime \prime \prime \prime}-\lambda^{4} X=\frac{k_{1} X\left(x_{0}\right.}{E J} \frac{\omega_{0}^{2}}{\omega_{0}^{2}-\omega^{2}} \delta\left(x-x_{0}\right), \\
& \omega_{0}^{2}=\frac{k_{1}}{m} .
\end{aligned}
$$

The general solution of Eq. (40) has the form

$$
\begin{aligned}
& X(x)=Q \sin \lambda x+S \sinh \lambda x+\frac{k_{1}}{2 E J \lambda^{3}} \frac{\omega_{0}^{2}}{\omega_{0}^{2}-\omega^{2}} X\left(x_{0}\right) \\
& \quad \times\left[\sinh \lambda\left(x-x_{0}\right)-\sin \lambda\left(x-x_{0}\right)\right] H\left(x-x_{0}\right)
\end{aligned}
$$

From Eqs. (40) and (36), we obtain

$$
\left\{\begin{array}{c}
Q \sin \lambda l+S \sinh \lambda l+\frac{k_{1}}{2 E J \lambda^{3}} \frac{\omega_{0}^{2}}{\omega_{0}^{2}-\omega^{2}} X\left(x_{0}\right) \\
\times\left[\sinh \lambda\left(l-x_{0}\right)-\sin \lambda\left(l-x_{0}\right)\right]=0, \\
-Q \sin \lambda l+S \sinh \lambda l+\frac{k_{1}}{2 E J \lambda^{3}} \frac{\omega_{0}^{2}}{\omega_{0}^{2}-\omega^{2}} X\left(x_{0}\right) \\
\times\left[\sinh \lambda\left(l-x_{0}\right)+\sin \lambda\left(l-x_{0}\right)\right]=0 .
\end{array}\right.
$$

By Eq. (41)

$$
X_{0}\left(x_{0}\right)=Q \sin \lambda x_{0}+S \sinh \lambda x_{0},
$$

hence from Eq. (42) we obtain the equations for eigenvalues

$$
\left|\begin{array}{l}
b_{11} b_{12} \\
b_{21} b_{22}
\end{array}\right|=0,
$$

where

$$
\begin{aligned}
b_{11} & =\sin \lambda l+\frac{k_{1}}{2 E J \lambda^{3}} \sin \lambda x_{0} \frac{\omega_{0}^{2}}{\omega_{0}^{2}-\omega^{2}} \\
\times & {\left[\sinh \lambda\left(l-x_{0}\right)-\sin \lambda\left(l-x_{0}\right)\right], } \\
b_{12} & =\sinh \lambda l+\frac{k_{1}}{2 E J \lambda^{3}} \sinh \lambda x_{0} \frac{\omega_{0}^{2}}{\omega_{0}^{2}-\omega^{2}} \\
\quad \times & {\left[\sinh \lambda\left(l-x_{0}\right)-\sin \lambda\left(l-x_{0}\right)\right], } \\
b_{21} & =-\sin \lambda l-\frac{k_{1} \sin \lambda x_{0}}{2 E J \lambda^{3}} \sin \lambda x_{0} \frac{\omega_{0}^{2}}{\omega_{0}^{2}-\omega^{2}} \\
\quad \times & {\left[\sinh \lambda\left(l-x_{0}\right)+\sin \lambda\left(l-x_{0}\right)\right], } \\
b_{22} & =\sinh \lambda l+\frac{k_{1} \sinh \lambda x_{0}}{2 E J \lambda^{3}} \sin \lambda x_{0} \frac{\omega_{0}^{2}}{\omega_{0}^{2}-\omega^{2}} \\
\quad \times & {\left[\sinh \lambda\left(l-x_{0}\right)+\sin \lambda\left(l-x_{0}\right)\right] . }
\end{aligned}
$$

The form of the terms of determinant Eq. (44) shows that $\lambda_{n}=\lambda_{n}\left(k_{1}\right)$. If it turns out that

$$
\lambda_{n}\left(k_{1}\right)<\frac{\pi n}{l},
$$

then the adopted model presented in Fig. 1 is correct.

We can summarize the conclusions as follows:

- Replacing the formula (2) with Eq. (7) turned out to be a more general approach to problems of this kind. The obtained equation (24) of eigenfunctions includes the existing torque and transverse force at the point of fracture $x_{0}$ and the local deformation of the beam axis in the neighbourhood of $x_{0}$. 
- If $\gamma_{1} \rightarrow-1$, then equation (24) becomes the equation of the beam with a point joint at the point $x_{0}[5]$.

- In the existing literature of this subject numerical calculations are executed for $n=1$. Demonstration of the decreasing character of $\lambda_{n}\left(\gamma_{1}\right)$ and $\lambda_{n}(k)$ in relation to $\frac{\pi n}{l}$, at least for $n=1$, would prove that the models described in the present work are correct and equivalent to each other.

\section{Conclusions}

This paper presents analytical solutions of the free vibration problems for a simply supported beam with local increase of stiffness. Knowing the eigenfuctions and boundary conditions we can determine a system of algebraic equations for the eigenvalues that are different from the eigenvalues of the beam without local stress distribution. The advantage of this approach consists in compact mathematical description. Results can be useful for testing the approximate solutions obtained by other methods.

Since the equations of eigenvalues have been derived, the goal of the study has been achieved. The models under consideration will be equivalent if the roots of the equations are identical or differ slightly. On the other hand, the models under consideration will be correct if their zero points (at least the first few) are smaller than the eigenvalues of the beam of a constant diameter, $\frac{\pi n}{l}$ ( $n=1,2, \ldots l$ - beam length).

The answers to the questions posed above may be obtained either theoretically or with the help of computed analysis for given parameters. These answers will be the subject of a subsequent study.

\section{References}

[1] S. Kasprzyk, Acta Phys. Pol. A 121, 981 (2012).

[2] M. Wiciak, S. Kasprzyk, Opuscula Math. 27, 245 (2007).

[3] M. Jabłonski, A. Ozga, T. Korbiel, P. Pawlik, Acta Phys. Pol. A 119, 971 (2011).

[4] F.P. Beer, E.R. Johnson (Jr.), Mechanics for Engineers, Cliffs, New Jersey 1977.

[5] S. Kasprzyk, R. Marczuk, Acta Physica Polonica A 121, A-191 (2012).

[6] S. Kasprzyk, Dynamics of Continuous Systems. University's Scripts, Wydawnictwo AGH, Kraków 1988, (in Polish).

[7] P. Antosik, J. Mikulski, R. Sikorski, Theory of Distributions; the Sequental Approach, Elsevier Sci. Publ., New York 1973.

[8] J. Mikusiński, R. Sikorski, Elementary Theory of Distribution, PWN, Warszawa 1964.

[9] L. Schwartz, Mathematics for Physical Sciences, PWN Warszawa 1984.

[10] S. Kaliski, Technical Mechanics. Waves and Vibrations, PWN, Warszawa 1986. 